\title{
Dispositif de mesure de la conductivité thermique effective longitudinale de torons adapté à différents degrés de carbonisation
}

\section{Device for measuring the longitudinal effective thermal conductivity of strands adapted to different stages of carbonization}

\author{
Baptiste BOUYER ${ }^{1}$, Xavier TARDIF ${ }^{2}$, Célia MERCADER ${ }^{3}$, Didier DELAUNAY ${ }^{4}$ \\ ${ }^{1}$ LTeN, UMR 6607, Université de Nantes, CNRS, Nantes, France, baptiste.bouyer@univ-nantes.fr \\ ${ }^{2}$ Institut de Recherche Technologique (IRT) Jules Verne, Bouguenais, France, xavier.tardif@irt-jules-verne.fr \\ ${ }^{3}$ Plateforme CANOE, Pessac, France, mercader@plateforme-canoe.com \\ ${ }^{4}$ LTeN, UMR 6607, Université de Nantes, CNRS, Nantes, France, didier.delaunay@univ-nantes.fr
}

\begin{abstract}
RÉSUMÉ. Un dispositif de mesure de conductivité thermique longitudinale de torons de fibres a été mis au point et modélisé par éléments finis. Le modèle numérique a permis de minimiser les pertes radiatives. Des mesures de conductivité thermique ont été réalisées sur trois échantillons étalons représentatifs de la gamme de mesure et valident ce dispositif de $0,1 \mathrm{~W} \cdot \mathrm{m}^{-1} \cdot \mathrm{K}^{-1}$ à $100 \mathrm{~W} \cdot \mathrm{m}^{-1} \cdot \mathrm{K}^{-1}$ ainsi que sur des échantillons constitués de fibres de viscose et de fibres de carbone T300 ce qui a permis de valider le dispositif pour la caractérisation des fibres de précurseur aux fibres de carbone.

ABSTRACT. A device for for fibers axial thermal conductivity measurement has been developed and modeled by finite elements. The numerical model made it possible to minimize the radiative losses. Thermal conductivity measurements have been performed on three standard samples representative of the measurement range and validate this device from $0.1 \mathrm{~W} \cdot \mathrm{m}^{-1} \cdot \mathrm{K}^{-1}$ to $100 \mathrm{~W} \cdot \mathrm{m}^{-1} \cdot \mathrm{K}^{-1}$ as well as on samples made up of viscose fibers and T300 carbon fibers, which validated the device for the characterization of fiber strands from precursor fibers to carbon fibers.
\end{abstract}

MOTS-CLÉS. Conductivité thermique, Fibres, Torons, Plaque chaude gardée.

KEYWORDS. Thermal conductivity, Fibers, Strands, Guarded hot plate.

\section{Nomenclature}

L longueur, $\mathrm{m}$

$P \quad$ puissance, $\mathrm{W}$

$T$ température, $\mathrm{K}$ et ${ }^{\circ} \mathrm{C}$

Symboles grecs

$\Delta \quad$ différence

$\Phi \quad$ flux de chaleur, W

$\lambda \quad$ conductivité thermique, $W \cdot m^{-1} \cdot K^{-1}$

Indices et exposants

chaud système de chauffage cond

conduction

ech échantillon

eff effective

elec électrique

froid système de refroidissement

num numérique

paroi paroi

rad radiatif

regul régulation

théo théorique

$x, y, z \quad$ directions 


\section{Introduction}

Les fibres sont de plus en plus utilisées notamment comme renforts dans les matériaux composites. Selon la nature de ces fibres, la littérature fait état de conductivités thermiques très basses avec un ordre de grandeur de $0,1 \mathrm{~W} \cdot \mathrm{m}^{-1} \cdot \mathrm{K}^{-1}$ pour les fibres les plus isolantes allant jusqu'à des valeurs très hautes, pouvant atteindre $100 \mathrm{~W} \cdot \mathrm{m}^{-1} \cdot \mathrm{K}^{-1}$ [DUP 08] ou plus pour les fibres de carbone les plus conductrices. Les fibres sont rassemblées pour former des torons. La conductivité thermique effective des torons de fibres dépend non seulement du matériau des fibres mais aussi de leur morphologie [REN 02] : nombre, diamètre, orientation (torsadé ou non), continuité des fibres, etc. Ainsi la caractérisation thermique des torons est une étape critique dans l'évaluation des propriétés thermiques des matériaux composites.

S'il existe aujourd'hui de nombreuses méthodes pour mesurer la conductivité thermique des fibres micrométriques avec la méthode $3 \omega$ [MIS 18] ou encore la méthode flash [DEM 09], seules quelques méthodes [PIR 87], [GAL 00], [CAN 19] permettent de mesurer la conductivité thermique de torons mais sont réservées aux fibres de conductivité thermique supérieure à $10 \mathrm{~W} \cdot \mathrm{m}^{-1} \cdot \mathrm{K}^{-1}$.

Compte tenu de la spécificité des fibres, un moyen de mesure direct de conductivité thermique dédié aux fibres semble pertinent.

Nous présentons ici un nouvel appareil permettant de mesurer la conductivité thermique longitudinale de torons de fibres sur trois ordres de grandeur de $0,1 \mathrm{~W} \cdot \mathrm{m}^{-1} \cdot \mathrm{K}^{-1}$ à $100 \mathrm{~W} \cdot \mathrm{m}^{-1} \cdot \mathrm{K}^{-1}$ soit de la fibre de précurseur à la fibre de carbone.

\section{Méthodologie}

\subsection{Principe de la méthode}

La méthode est inspirée de la technique de la plaque chaude gardée. La résistance thermique d'un échantillon est obtenue par la loi de Fourier en 1D en régime permanent. De cette résistance thermique est déduite la conductivité thermique du matériau connaissant les dimensions de l'échantillon. Généralement l'échantillon est une plaque soumise à un gradient de température unidirectionnel, le dispositif étant isolé thermiquement, tel que les pertes de chaleur latérales soient négligeables. La détermination de la conductivité thermique, $\lambda$ repose alors sur la mesure de la différence de température $\Delta \mathrm{T}$ entre les faces séparées d'une distance $\mathrm{L}$ et la mesure du flux de chaleur $\Phi$ traversant l'échantillon de section droite $\mathrm{S}$, correspondant à la puissance électrique $\mathrm{P}_{\text {élec }}$ consommée de l'élément chauffant.

Dans le cas de la plaque chaude gardée adaptée aux torons de fibres, l'échantillon est constitué de quelques torons identiques alignés, de plusieurs centimètres de longueur pour un diamètre millimétrique. La surface latérale de l'échantillon est donc grande devant sa section. Aussi une attention particulière doit être apportée aux pertes de chaleur, notamment radiatives, dans ce dispositif.

\subsection{Dispositif expérimental}

Le dispositif est représenté schématiquement sur la Figure 1. Les torons de fibres (2) sont maintenus tendus verticalement entre un système de chauffage (1) et un système de refroidissement (4). La température de l'échantillon est mesurée avec des thermocouples de type K. 


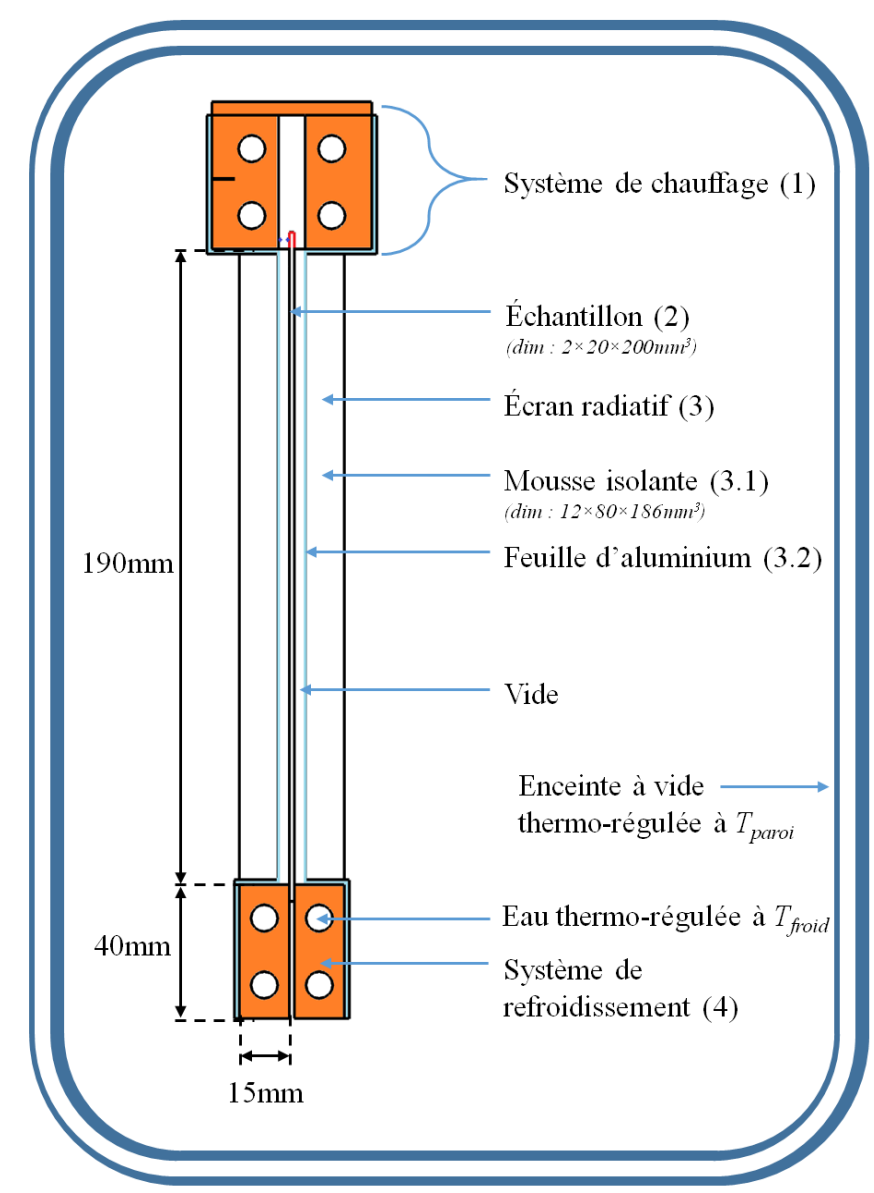

a) Dispositif entier (enceinte à vide non à l'échelle)

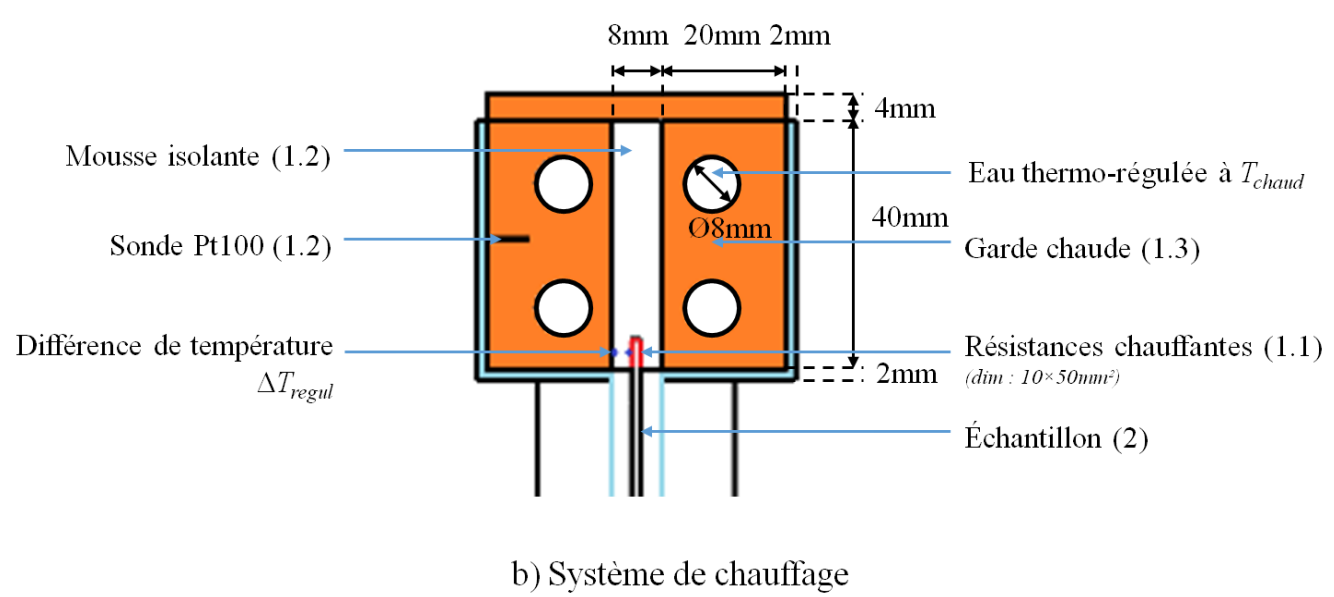

Figure 1. a) Schéma du dispositif entier, b) Schéma du système de chauffage.

Le système de chauffage (1) est constitué de résistances chauffantes (1.1) dont la puissance électrique consommée est mesurée et entre lesquelles est maintenu l'échantillon (2). Cet ensemble est isolé thermiquement par une mousse (1.2) de conductivité thermique $0,03 \mathrm{~W} \cdot \mathrm{m}^{-1} \cdot \mathrm{K}^{-1}$ le tout étant entouré par une garde chaude (1.3) constituée d'échangeurs de chaleur en cuivre dans lesquels circule de l'eau régulée à la température $T_{\text {chaud. }}$ Le système de refroidissement (4) est constitué d'échangeurs de chaleur en cuivre dans lesquels circule également de l'eau régulée à la température $T_{\text {froid }}$, telle que $\mathrm{T}_{\text {chaud }}$ soit supérieure à $\mathrm{T}_{\text {froid }}$.

Un écran radiatif (3) fixé aux échangeurs est constitué de mousse isolante (3.1) et de feuilles d'aluminium adhésives (3.2) entourant l'échantillon pour limiter les échanges de chaleur par rayonnement entre l'échantillon et son environnement. Seules les surfaces de l'écran en regard avec l'échantillon sont munies d'aluminium. Le dispositif est placé dans une enceinte elle-même régulée en 
température à $\mathrm{T}_{\text {paroi }}$ et sous vide primaire à $10^{-4}$ mbar pour éviter les pertes de chaleur par convection entre l'échantillon et l'air ambiant. Le vide permet également de retirer le gaz interstitiel présent dans les torons, ainsi la conductivité thermique mesurée est la conductivité thermique du matériau seul et non la conductivité thermique effective. L'eau thermorégulée circulant dans chacune des gardes provient de bains thermostatés à circulation disposant de leur propre système de régulation PID. Les résistances chauffantes (1.1) présentent chacune une résistance électrique de $60,3 \Omega$ et sont connectées en parallèle à une source de courant continu Keithley 6221 régulée par PID. Les paramètres de la régulation sont évalués avant chaque mesure. Le système de régulation ainsi que l'acquisition des températures ont été réalisés en utilisant le logiciel LabVIEW ${ }^{\mathrm{TM}}$ (National Instrument Corp.)

\subsection{Modélisation numérique du dispositif}

Un modèle numérique 3D de l'ensemble du dispositif représenté sur la Figure 1 a été réalisé sur le logiciel COMSOL Multiphysics ${ }^{\circledR}$ pour appréhender les pertes de chaleur par rayonnement entre l'échantillon et son environnement, rappelons que l'on travaille sous un vide de $10^{-4}$ mbar et que les pertes convectives sont négligeables. Les transferts radiatifs sont modélisés par la méthode des radiosités [COM 5.3] et les facteurs de forme déterminés par la méthode des hémicubes [COH 85] avec une résolution de 512. Pour chaque élément de maillage appartenant à une surface diffusive, le demiespace avant est projeté sur un hémicube centré sur l'élément considéré. Une résolution de 512 signifie que chaque face de l'hémicube est discrétisée en $512^{2}$ pixels. Le rapport des pixels recouverts par la projection d'une surface sur le nombre de pixels total donne le facteur de forme [COM 5.3]. L'échantillon modélisé est une bande de PMMA de $200 \mathrm{~mm}$ de longueur, $20 \mathrm{~mm}$ de largeur et de 1,5 $\mathrm{mm}$ d'épaisseur. L'epaisseur caractéristique de l'échantillon, soit le rapport surface sur périmetre, est $\mathrm{e}_{\mathrm{c}}=6.98 \cdot 10^{-4} \mathrm{~m}$. La conductivité thermique du PMMA utilisée dans le modèle est de $0,18 \mathrm{~W} \cdot \mathrm{m}^{-1} \cdot \mathrm{K}^{-1}$. Cet échantillon sera utilisé ensuite comme étalon pour caractériser le dispositif expérimental. On impose les conditions aux limites suivantes : température de paroi imposée sur les parois extérieures de l'enceinte à vide, flux de chaleur convectif imposé sur les parois des canaux des échangeurs de chaleur dans lesquels circulent de l'eau imposée à $30^{\circ} \mathrm{C}$ pour le système de chauffage et $20^{\circ} \mathrm{C}$ pour le système de refroidissement. L'ensemble des surfaces libres du dispositif ainsi que les surfaces intérieures de l'enceinte sont considérées comme surfaces diffusives : deux surfaces en regard à des températures différentes échangent de la chaleur par rayonnement. L'ensemble est à température ambiante à l'instant initial. Le maillage est constitué d'environ $2.10^{5}$ éléments tétraédriques.

L'étude est réalisée en régime permanent. L'utilisation du bouclier radiatif, différentes températures de paroi et différentes longueurs d'échantillons ont été testées. Les configurations des simulations et leurs résultats sont rapportés dans le Tableau 1. Le flux de chaleur par conduction théorique est obtenu en négligeant toutes pertes de chaleur. 


\begin{tabular}{|c|c|c|c|c|c|c|}
\hline $\mathbf{N}^{\circ}$ de simulation & $\# 1$ & \#2 & \#3 & $\# 4$ & \#5 & \#6 \\
\hline Ecran radiatif & Non & Oui & Oui & Oui & Oui & Oui \\
\hline $\mathbf{T}_{\text {paroi }} /{ }^{\circ} \mathrm{C}$ & 25 & 25 & 20 & 30 & 25 & 25 \\
\hline $\mathrm{L}_{\mathrm{eff}, \text { éch }} / \mathrm{e}_{\mathrm{c}}\left(\mathrm{L}_{\mathrm{eff}, \text { éch }} / \mathrm{mm}\right)$ & \multicolumn{4}{|c|}{$272(190)$} & $201(140)$ & $129(90)$ \\
\hline$\Phi_{\text {cond théo }} / \mathrm{W}$ & \multicolumn{4}{|c|}{$-2,84.10^{-4}$} & $-3,86.10^{-4}$ & $-6,0.10^{-4}$ \\
\hline$\Phi_{\text {rad,num }} / W$ & $-2,59.10^{-4}$ & $8,71.10^{-6}$ & $-5,74.10^{-4}$ & $5,61.10^{-4}$ & $2,21.10^{-5}$ & $5,47.10^{-5}$ \\
\hline$\left|\Phi_{\text {rad num }} / \Phi_{\text {cond z num }}\right|$ & $93 \%$ & $3,1 \%$ & $203 \%$ & $198 \%$ & $5,8 \%$ & $9,3 \%$ \\
\hline | $\Phi_{\text {cond z num }} / \Phi_{\text {cond théo }} \mid$ & $98 \%$ & $98,4 \%$ & $99,6 \%$ & $99,4 \%$ & $98,7 \%$ & $98,2 \%$ \\
\hline$\left|\Phi_{\text {cond } \mathrm{x}} / \Phi_{\text {cond } \mathrm{z}}\right|$ & $0,0 \%$ & $1,2 \%$ & $0,1 \%$ & $0,1 \%$ & $0,0 \%$ & $0,3 \%$ \\
\hline$\left|\Phi_{\text {cond y }} / \Phi_{\text {cond z }}\right|$ & $16 \%$ & $1,6 \%$ & $2,7 \%$ & $2,6 \%$ & $0,6 \%$ & $0,6 \%$ \\
\hline
\end{tabular}

Tableau 1. Simulations numériques du dispositif : paramètres et résultats.

Les champs de température obtenus avec et sans écran radiatif pour une température de paroi de $25^{\circ} \mathrm{C}$ sont représentés sur la Figure 2. On peut noter que le gradient de température dans l'échantillon est unidirectionnel uniquement en présence de l'écran radiatif. Dans le cas contraire, l'échantillon est en grande partie à la température de paroi : les transferts radiatifs entre l'échantillon et la paroi ne sont pas négligeables et atteignent le même ordre de grandeur que le flux de chaleur par conduction théorique, ce qui invaliderait le principe du dispositif. La présence de l'écran radiatif est donc nécessaire mais non suffisante pour garantir des transferts radiatifs négligeables. En effet, en présence de l'écran les pertes radiatives peuvent atteindre le double du flux de chaleur par conduction recherché lorsque la température de paroi est égale à la température de l'un des échangeurs (simulation \#3 et \#4). En revanche les pertes de chaleur par rayonnement sont minimisées lorsque la température de paroi est égale à la température moyenne des systèmes de chauffage et de refroidissement (simulation \#2).

Champs de température

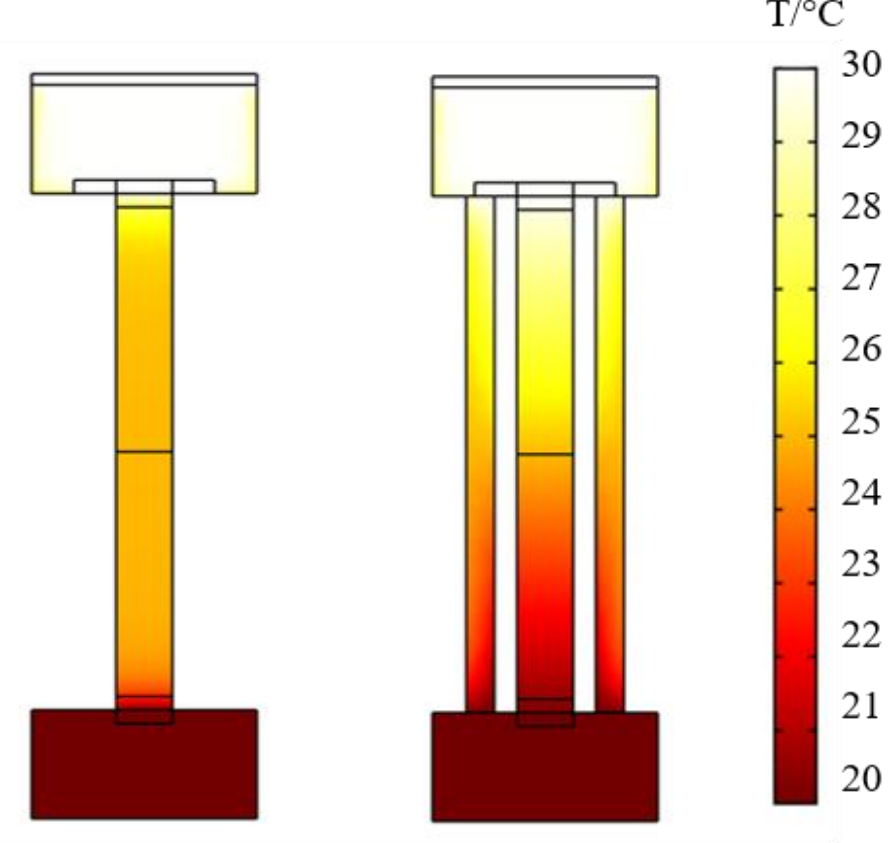

a) Sans écran radiatif

b) Avec écran radiatif

Figure 2. Champ de température dans le dispositif :

a) sans écran radiatif et b) avec écran radiatif 
Les simulations réalisées montrent également l'influence la longueur de l'échantillon (simulations \#2 \#5 et \#6) : en diminuant la longueur de l'échantillon, la résistance thermique de l'échantillon diminue également conduisant à une augmentation du flux de chaleur par conduction. Dans le même temps, le flux de chaleur radiatif augmente également, ce qui peut être attribué à l'effet de bords. La diminution de la longueur de l'échantillon entraîne une augmentation du facteur de forme géométrique entre les systèmes de chauffage ou de refroidissement et l'échantillon et donc à une augmentation des transferts de chaleur aux extrémités de l'échantillon. Les rapports des flux de chaleur radiatif et conductif présentés dans le Tableau 1 indiquent que les pertes radiatives augmentent plus vite que le flux conductif, un échantillon plus élancé (dont l'élancement, ou le rapport longueur sur épaisseur caractéristique, est plus grand) est donc préférable.

Un échantillon plus élancé est également avantageux pour d'autres raisons: il permet une instrumentation thermique plus facile et plus précise de l'échantillon puisque le gradient de température est plus faible et donc la mesure de température moins sensible à l'emplacement des capteurs tels que les thermocouples. Enfin, une plus grande résistance thermique de l'échantillon rend les résistances de contact, qui ne dépendent pas de la longueur de l'échantillon, relativement plus faibles et contribuent ainsi à les négliger. Des mesures seront effectuées sur des échantillons de longueur différente dans la partie 3 pour s'assurer que les résistances de contact n'affectent pas le résultat.

\subsection{Détermination de la conductivité thermique longitudinale}

La température du système de chauffage Tchaud est imposée par la garde. Les résistances chauffantes sont régulées en courant de telle sorte à annuler la différence de température $\Delta$ Tregul entre les résistances chauffantes et la garde. Une différence de température nulle indique que le système de chauffage est isotherme. Ainsi lorsque le régime permanent est atteint, puissance électrique consommée par les résistances chauffantes ne varie plus, le flux de chaleur traversant l'échantillon correspond alors à la chaleur dissipée par les résistances chauffantes et donc à leur consommation électrique.

\section{Résultats}

\subsection{Validation expérimentale du dispositif}

Des mesures ont été réalisées sur trois échantillons représentatifs de la gamme de mesure recherchée. Le premier est une bande de PMMA de dimension $1,5 \mathrm{~mm}$ x $20 \mathrm{~mm}$ x $200 \mathrm{~mm}$, le second une bande d'inox 316 de dimension $2 \mathrm{~mm} \times 20 \mathrm{~mm}$ x $200 \mathrm{~mm}$ et le troisième est une bande d'aluminium 2017A de même dimension que le second échantillon. Une attention particulière est portée sur la régulation de la température des parois de l'enceinte, devant être égale à la moyenne des températures des systèmes de chauffage et de refroidissement. La conductivité thermique obtenue expérimentalement $\lambda_{\exp }$ est comparée à la conductivité thermique du matériau correspondant trouvé dans la littérature [ASM 19], [RID 09] $\lambda_{\text {lit }}$ dans le Tableau 2. La conductivité thermique du PMMA est extraite de la référence [RID 09]. Elle dépend de la façon dont l'échantillon est obtenu (coulé ou extrudé) mais une valeur moyenne de $0.19 \pm 0,01 \mathrm{~W} \cdot \mathrm{m}^{-1} \cdot \mathrm{K}^{-1}$ peut être admise. 


\begin{tabular}{|c|c|c|c|}
\hline Matériau & PMMA & Inox 316 & Aluminium 2017A \\
\hline$\lambda_{\exp } /\left(\mathbf{W} \cdot \mathbf{m}^{-\mathbf{1}} \cdot \mathbf{K}^{-\mathbf{1}}\right)$ & 0,203 & 16,19 & 133,4 \\
\hline$\lambda_{\text {lit }} /\left(\mathbf{W} \cdot \mathbf{m}^{-\mathbf{1}} \cdot \mathbf{K}^{-\mathbf{1}}\right)$ & $0,19[\mathrm{RID} 09]$ & $16,2[\mathrm{ASM} 19]$ & $134[\mathrm{ASM} 19]$ \\
\hline
\end{tabular}

Tableau 2. Résultats des mesures de conductivité thermique sur matériaux massifs de conductivité thermique connue

Une grande précision est obtenue pour les échantillons métalliques puisqu'on obtient un écart relatif de $0.06 \%$ pour l'inox et de $0.45 \%$ pour l'aluminium. En revanche un écart relatif plus important de $6.8 \%$ est obtenu pour l'échantillon le moins conducteur de chaleur, comme on pouvait s'y attendre, les pertes radiatives étant relativement plus grandes par rapport au flux de chaleur par conduction qui traverse l'échantillon.

\subsection{Résultats expérimentaux sur fibres}

L'appareil étant validé sur des échantillons massifs, des mesures ont ensuite été effectuées sur des torons de fibres. Quatre mesures ont été effectuées sur des échantillons constitués de 10 torons de 6000 fibres de carbone T300 de $6 \mu \mathrm{m}$ de diamètre et de $200 \mathrm{~mm}$ de hauteur pour les 2 premières mesures et de $150 \mathrm{~mm}$ pour les deux autres. Les résultats montrent que la conductivité thermique ne change pas avec la longueur de l'échantillon et confirment que les résistances de contact thermique sont négligeables. Les résultats obtenus sont présentés sur la Figure 3 et comparés aux conductivités thermiques trouvées dans la littérature [MIS 18], [TOR 18], [CYT 12], [YAM 96], [KAW 02], [WU 12]. Les conductivités thermiques obtenues expérimentalement avec le nouveau dispositif sont comprises entre $8,4 \mathrm{~W} \cdot \mathrm{m}^{-1} \cdot \mathrm{K}^{-1}$ et $8,7 \mathrm{~W} \cdot \mathrm{m}^{-1} \cdot \mathrm{K}^{-1}$ et sont en bon accord avec la valeur moyenne des conductivités thermiques disponibles dans la littérature (valeur moyenne de $8,37 \mathrm{~W} \cdot \mathrm{m}^{-1} \cdot \mathrm{K}^{-1}$ ), ce qui valide l'utilisation de ce nouveau dispositif de mesure de la conductivité thermique longitudinale effective de torons de fibres conductrices. La valeur moyenne n'est certes pas un très bon estimateur mais compte tenu de la dispersion des résultats, y compris entre les différents constructeurs [TOR 18], [CYT 12] il est difficile de trouver une référence.

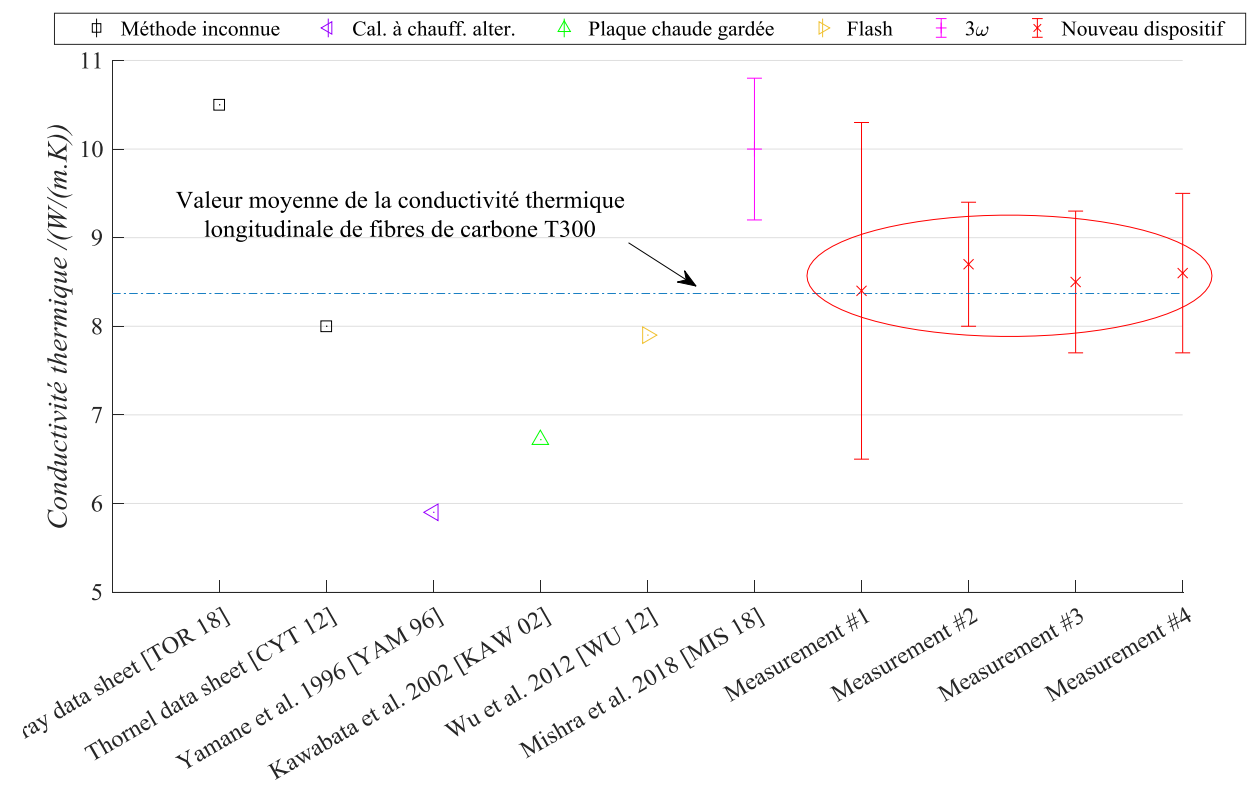

Figure 3. Conductivité thermique axiale des fibres de carbone T300 : état de l'art et mesures 
Enfin, des mesures ont été réalisées sur des fibres isolantes de viscose utilisée notamment comme précurseur pour la fabrication de fibre de carbone. Le nombre de torons utilisés, la différence de température appliquée, la puissance électrique mesurée et la conductivité thermique obtenue sont présentés dans le Tableau 3. Kawabata et al. [KAW 02] ont également mesuré la conductivité thermique de fibres de viscose et obtiennent une valeur de $1,077 \mathrm{~W} \cdot \mathrm{m}^{-1} \cdot \mathrm{K}^{-1}$, ce qui est très proche des valeurs expérimentales obtenues (un écart relatif de 5.0\% est obtenu entre la valeur moyenne des résultats expérimentaux et la valeur de Kawabata et al.). Le nouveau dispositif est donc également validé pour les mesures de conductivité thermique de fibres isolantes.

\begin{tabular}{|c|c|c|c|}
\hline Numéro de mesure & $\mathbf{\# 1}$ & $\mathbf{\# 2}$ & $\mathbf{\# 3}$ \\
\hline Nombre de fibres & $22 * 1 \mathrm{k}$ & $58 * 1 \mathrm{k}$ & $58 * 1 \mathrm{k}$ \\
\hline$\Delta \mathbf{T} / \mathbf{K}$ & 8,65 & 30,01 & 29,93 \\
\hline $\mathbf{P}_{\text {élec }} / \mathbf{W}$ & $1,33 \cdot 10^{-4}$ & $1,08.10^{-3}$ & $1,16.10^{-3}$ \\
\hline$\lambda_{\text {exp }} /\left(\mathbf{W} \cdot \mathbf{m}^{-1} \cdot \mathbf{K}^{-\mathbf{1}}\right)$ & $1.08 \pm 0,07$ & $0,96 \pm 0,04$ & $1.03 \pm 0,05$ \\
\hline
\end{tabular}

Tableau 3. Résultats des mesures de conductivité thermique axiale sur des fibres de viscose

\section{Conclusion}

Un dispositif de mesure de conductivité thermique longitudinale de torons de fibre inspiré de la méthode de la plaque chaude gardée a été mis au point et modélisé numériquement par éléments finis, réalisé, testé et validé. Le modèle numérique a permis de vérifier que les pertes de chaleur par rayonnement sont minimisées si la température de paroi est égale à la température moyenne des systèmes de chauffage et de refroidissement en présence d'un écran radiatif. Des mesures expérimentales ont été conduites sur trois échantillons étalons représentatifs de la gamme de mesure du dispositif et valident le dispositif sur la gamme de conductivité thermique recherchée : de $0,1 \mathrm{~W} \cdot \mathrm{m}^{-1} \cdot \mathrm{K}^{-1}$ à $100 \mathrm{~W} \cdot \mathrm{m}^{-1} \cdot \mathrm{K}^{-1}$. Ensuite des mesures ont été réalisées sur des torons de fibres isolantes (viscose) et conductrices (T300) et comparées à la littérature ce qui a permis de valider le dispositif pour les mesures de conductivité thermique de torons de fibres, des fibres de précurseur aux fibres de carbone.

\section{Bibliographie}

[DUP 08] DUPUPET, G., "Fibres de carbone", Tech. l'ingénieur, p. 1-19, 2008.

[REN 02] RENGASAMY, R. S. et KAWABATA, S., "Computation of thermal conductivity of fibre from thermal conductivity of twisted yarn", Indian J. Fibre Text. Res., n 27, p. 342-345, 2002.

[MIS 18] MishrA, K. ET AL., "Thermal properties measurement of single fiber with the 3 omega method", in 12th International Conference on Composite Science and Technology, 2018.

[DEM 09] DEMKO, M. T. ET AL., "Application of the thermal flash technique for low thermal diffusivity micro/nanofibers", Rev. Sci. Instrum., n 80, p. 1-3, 2009.

[PIR 87] PIRAUX, L., ISSI, J.-P., et COOPMANS, P., "Apparatus for thermal conductivity measurements of thin fibres", Measurement, $\mathrm{n}^{\circ}$ 5, p. 2-5, 1987.

[GAL 00] GaLlego, N. C. ET AL., "Thermal conductivity of ribbon-shaped carbon fibers", Carbon N. Y., $\mathrm{n}^{\circ}$ 38, p. 1003-1010, 2000. 
[CAN 19] Candadai, A. A., Weibel, J. A., et Marconnet, A. M., "A Measurement Technique for Thermal Conductivity Characterization of Ultra-High Molecular Weight Polyethylene Yarns Using High-Resolution Infrared Microscopy", in 2019 18th IEEE Intersociety Conference on Thermal and Thermomechanical Phenomena in Electronic Systems (ITherm), 2019, p. 490-497, .

[COM 5.3] "COMSOL 5.3 Heat Transfer Module User's Guide".

[COH 85] Cohen, M. F. et GreEnBerg, D. P., "The hemi-cube, a radiosity solution for complex environments", in SIGGRAPH'85, 1985, p. 31-40, .

[ASM 19] ASM AerospaCe SPECIFICATION Metals INC., "asm.matweb.com", 2019. Disponible à : http://www.aerospacemetals.com/aluminum-distributor.html. Consulté le : 02.04.2019.

[RID 09] RIDES, M. ET AL., "Intercomparison of thermal conductivity and thermal diffusivity methods for plastics", Polym. Test., $\mathrm{n}^{\circ}$ 28, p. 480-489, 2009.

[TOR 18] TORAYCA, "T300 DATA SHEET", 2018.

[CYT 12] CYTEC ENGINEERED MATERIALS, "THORNEL® T-300 PAN-BASED FIBER", 2012.

[YAM 96] YAMANE, T. ET AL., "Thermal diffusivity measurement of single fibers by an ac calorimetric method", J. Appl. Phys., ${ }^{\circ}$ 80, p. 4358-4365, 1996.

[KAW 02] KaWABAta, S. et Rengasamy, R. S., "Thermal conductivity of unidirectional fibre composites made from yarns and computation of thermal conductivity of yarns", Indian J. Fibre Text. Res., n ${ }^{\circ}$ 27, p. 217-223, 2002.

[WU 12] WU, G.-P. ET AL., "Carbon layer structures and thermal conductivity of graphitized carbon fibers", J. Mater. Sci., n ${ }^{\circ}$ 47, p. 2882-2890, 2012.

\section{Remerciements}

Dans un premier temps, nous remercions Nicolas Lefevre pour la pertinence de ses propos et d'autre part Arnaud Arrivé et Julien Aubril pour leur implication dans la réalisation de ce dispositif, tous trois travaillant pour le Service Études et Fabrication (SEF) du LTeN.

Ces travaux font partie du projet FORCE piloté par l'IRT Jules Verne (Institut français de recherche technologique des technologies de fabrication avancées pour les structures composites, métalliques et hybrides). 